\title{
Odor-searching Robot with Insect-behavior-based Olfactory Sensor
}

\author{
Junji Horibe, ${ }^{1}$ Noriyasu Ando, ${ }^{1,2 *}$ and Ryohei Kanzaki ${ }^{1}$ \\ ${ }^{1}$ Research Center for Advanced Science and Technology, The University of Tokyo, \\ 4-6-1 Komaba, Meguro-ku, Tokyo 153-8904, Japan \\ ${ }^{2}$ Department of Systems Life Engineering, Maebashi Institute of Technology, \\ 460-1 Kamisadori-cho, Maebashi, Gunma 371-0816, Japan
}

(Received March 21, 2021; accepted September 2, 2021)

Keywords: olfactory sensor, silkmoth, odor searching, machine learning, robot

Extremely sensitive odor sensors are required for odor searching in mobile robots. The male silkmoth (Bombyx mori) is a candidate biosensor because of its high sensitivity to the conspecific sex pheromone with stereotypic searching behavior. Furthermore, the odor preferences of silkmoths can be modified using genetic tools. Therefore, techniques that can easily detect the odor response of silkmoths with high sensitivity have become important for odor detection and searching. In recent years, machine learning has been used to classify the behaviors of silkmoths to estimate the timing of odor reception. Therefore, it is possible to utilize a silkmoth's behavioral response as an olfactory sensor for robotic odor searching. In this work, we developed an odorsearching mobile robot with an odor sensing device based on a silkmoth's walking pattern. First, we collected behavioral data with and without odor stimuli. Then, we predicted the presence of an odor using a support vector machine. Finally, we implemented the sensing device in an odorsearching robot and demonstrated that the classifier performance was sufficient for a robot to localize an odor source by utilizing artificial searching algorithms. These results indicated the feasibility of an insect-behavior-based olfactory sensor for robotic odor searching.

\section{Introduction}

The practical application of mobile robots that can detect target odors and identify their source in a real environment is expected for security and rescue missions. To realize robotic odor searching, technologies such as odor detection, target odor identification, and odor source localization have been developed in the field of engineering. ${ }^{(1-4)}$ On the other hand, olfaction provides important sensory information for animals for survival and reproduction, such as for finding food, nests, and mating partners or avoiding predators and hazardous areas. Therefore, the olfactory sensory-motor system in animals has motivated engineers to develop bioinspired, biomimetic, ${ }^{(1-4)}$ and biohybrid robots ${ }^{(5,6)}$ to find odor sources.

The development of olfactory sensors that can detect a specified odor in a turbulent flow (common in the living environments of most organisms ${ }^{(7)}$ ) is one of the major challenges in *Corresponding author: e-mail: ando@maebashi-it.ac.jp https://doi.org/10.18494/SAM.2021.3369 
realizing robotic odor searching. There are three requirements for the olfactory sensors used in odor searching tasks. First, a sensor must detect a target odor away from an odor source (sensitivity). Second, the sensor must capture the temporal change in odor reception (temporal resolution) because a robot moves in an odor environment where the odor distribution is intermittent and changes with time. Third, the sensor must distinguish a specified target odor from numerous other odorants flowing in air (selectivity). The performance of conventional chemical sensors used in robotics, such as semiconductor metal oxide gas sensors, is inadequate in terms of these three requirements when they are compared with the odor sensing ability of animals. ${ }^{(1)}$ Insects are candidate model organisms for developing biosensors that meet all these requirements. ${ }^{(8)}$ The odor sensitivity of insect antennae, which are their olfactory organs, is extremely high. Flying insects can detect an odor far from an odor source. The male gypsy moth, Lymantria dispar, follows the plume of the conspecific sex pheromone over distances larger than $80 \mathrm{~m} .{ }^{(9)}$ The male pea moth, Cydia nigricana, can detect a pheromone $200 \mathrm{~m}$ from a source. ${ }^{(10)}$ In addition, the male silkmoth, Bombyx mori, initiates odor searching behavior using 170 sex-pheromone molecules. In terms of temporal properties, the yellow fever mosquito, Aedes aegypti, flies in the direction of a carbon dioxide source only $30 \mathrm{~ms}$ after coming in contact with a carbon dioxide plume. ${ }^{(11)}$ The time-resolved odor response can be evaluated by measuring the odor response of insect antennae using electroantennograms (EAGs). ${ }^{(12)}$ The EAGs of B. mori resolve the temporal structure of the pheromone oscillating up to $25 \mathrm{~Hz}{ }^{(13)}$ Furthermore, the EAG measurements of certain model insects (bees, moths, locusts, and cockroaches) resolve odor pulses more than $100 \mathrm{~Hz}$ with a response latency of less than $2 \mathrm{~ms} .^{(14)}$ Regarding selectivity, pheromone receptors are categorized as specialists with high selectivity for the conspecific sex pheromone even in the presence of background odors. Moreover, generalist-type olfactory receptors that respond to general odors have different selectivities for a particular odor. ${ }^{(15)}$ Therefore, insect antennae and EAG measurement have been used as olfactory sensors for detecting a specific pheromone ${ }^{(16,17)}$ and discriminating odors. ${ }^{(18-20)}$ Furthermore, insect antennae have also been used as olfactory sensors for mobile robots to evaluate searching algorithms in actual odor environments. ${ }^{(21-24)}$ However, EAG measurement has the disadvantage of being susceptible to electrical and mechanical noise. ${ }^{(25,26)}$ In particular, when EAG measurement is performed using mobile robots, the odor response decreases over time if isolated antennae are used. ${ }^{(22)}$

If the odor response measured from antennae can be directly linked to specific behaviors, it is possible to use an odor-triggered behavior as an index of the presence or absence of the odor with high sensitivity. ${ }^{(27)}$ This is the basis of insect-behavior-based biosensors [Fig. 1(a)]. ${ }^{(28)}$ The innate or learned preference or aversion to a specific odor has been tested by monitoring searching, feeding, and avoidance behaviors. ${ }^{(29,30)}$ In addition, portable detecting devices that perform training and testing using image processing or electromyography have been developed. For example, parasitic wasps, Microplitis croceipes, were trained using 3-octanone associated with food via classical conditioning, and their searching behavior in response to the odor was automatically detected from images. ${ }^{(31)}$ Behavior-based sensors are easy to set up and tolerant to electrical and mechanical noise compared with electrophysiological methods such as the use of EAGs and electromyograms. ${ }^{(32)}$ Thus, behavior-based sensors are suitable for use in odor- 


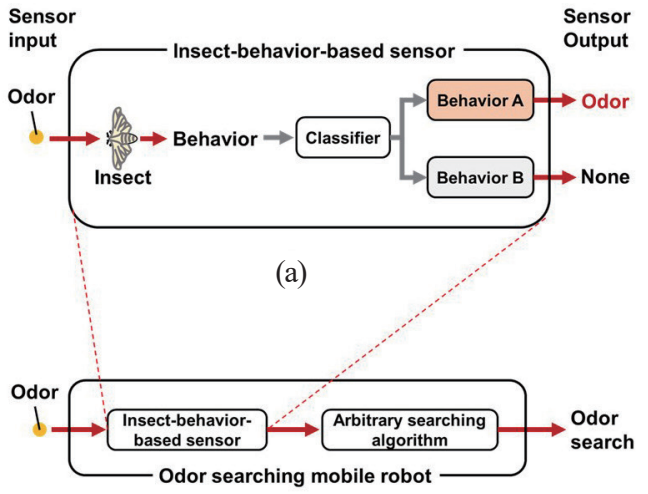

(b)

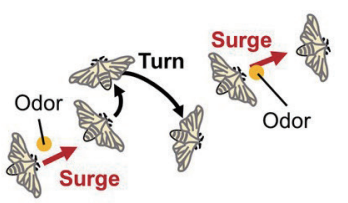

(c)

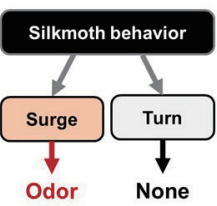

(d)

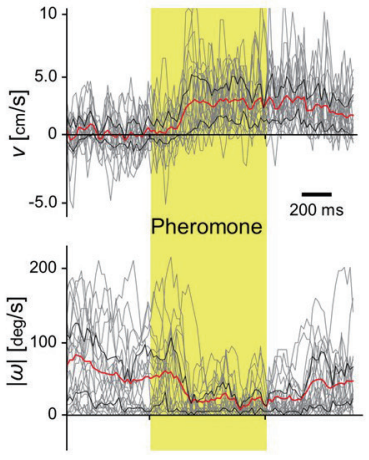

(e)

Fig. 1. (Color online) Insect-behavior-based olfactory sensor and odor searching behavior of a silkmoth. (a) Diagram of an insect-behavior-based olfactory sensor. If behavior A is directly linked to odor reception, the classified behavior can be regarded as a binary output of the presence or absence of odor. (b) Diagram of an odorsearching mobile robot equipped with an insect-behavior-based olfactory sensor. Arbitrary searching algorithms that accept a binary input can be used for the robot. (c) A silkmoth performs a surge during odor (sex pheromone) perception and consecutive turns when it fails to detect the odor. (d) Two behavioral states represent the presence or absence of odor. (e) Fore/aft (positive/negative) velocity $(v)$ and absolute angular velocity $(|\omega|)$ of a silkmoth in response to pheromone stimuli (yellow). Data obtained from 20 stimuli of a single stimulus sequence (gray), mean value (red), and 25 and $75 \%$ quantiles (black) are shown. Data are from Horibe et al. ${ }^{(36)}$

searching robots. However, there are no studies on odor-searching robots that use insectbehavior-based olfactory sensors. Therefore, the aim of this study is to develop an odor-searching mobile robot with an insect-behavior-based olfactory sensor [Fig. 1(b)].

We selected silkmoths (B. mori) for our work. Silkmoths have a stereotypic walking pattern for searching for female sex pheromones. ${ }^{(33)}$ They cannot fly and are quiescent without a pheromone stimulus. They start walking straight (surge) when they receive a pheromone stimulus and perform zigzagging turns when they lose the odor [Fig. 1(c)]. Therefore, these two behavioral states are useful indications of the presence or absence of the odor [Fig. 1(d)], which can be classified using machine learning techniques. The locomotion parameters that characterize the behavioral states are translational velocity (fore/aft) and turn angular velocity, which increase and decrease during pheromone reception, respectively [Fig. 1(e)]. Chew and Kurabayashi employed a hierarchical classification method to classify the behavior and predict the odor reception of silkmoths. ${ }^{(34)}$ Shigaki et al. measured the electromyograms of the flight muscles of silkmoths during the searching behavior, classified the behavioral states employing a support vector machine (SVM), and predicted pheromone reception. ${ }^{(35)}$ These studies demonstrate the potential use of silkmoth behaviors for olfactory sensors. However, the utilization of the silkmoth behavior as an olfactory sensor for an odor-searching mobile robot has not yet been reported. In our preliminary study, we proposed the concept of a mobile robot with a silkmoth-behavior-based olfactory sensor that employed the SVM classifier. ${ }^{(36)}$ In this study, we developed a mobile robot platform that enabled the training and testing of a behavior classifier, as well as odor searching by itself. We conducted odor searching experiments using a 
combination of the behavior-based olfactory sensor and artificial searching algorithms, and demonstrated the practicality of the behavior-based olfactory sensor in robotic odor searching tasks.

\section{Robot Hardware}

We developed an odor-searching robot equipped with a behavior-based olfactory sensor (Fig. 2). We used a commercially available mobile robot with dimensions of $215 \mathrm{~mm}$ (W) $\times$ $300 \mathrm{~mm}(\mathrm{D}) \times 100 \mathrm{~mm}(\mathrm{H})(10009$, Nexus Robot, Hong Kong, China) as a platform for odor sensing and searching. The hardware of the behavior-based odor sensor was composed of a spherical treadmill and an odor suction device. The appearance of the robot was similar to the silkmoth-driven mobile robot. ${ }^{(37)}$ However, the robot developed in this study used silkmoths only for detecting odors and implemented arbitrary searching algorithms. A male silkmoth was tethered at the dorsal thorax, and it walked on a polystyrene ball supported by air. An optical mouse (TS-0806, Trade Works, Tokyo, Japan) attached to the rear of an air-supported polystyrene ball (diameter of $50 \mathrm{~mm}$ ) measured the fore/aft velocity $(v)$ and left/right angular velocity $(\omega)$ of the silkmoth. The silkmoth responded to the conspecific female sex pheromone released from an odor source, which was suctioned by a DC fan (9BD12FC6-1, Sanyo, Osaka, Japan) through an intake with dimensions of $50 \mathrm{~mm}(\mathrm{H}) \times 100(\mathrm{~W}) \mathrm{mm}$. The locomotion data were acquired at a sampling rate of $50 \mathrm{~Hz}$ using an Arduino Uno with a USB host shield (SFE-DEV-09947, SparkFun Electronics, Boulder, CO, USA). Then, the data were sent to a laptop (Core i5 $7200 \mathrm{U}$ with 8 GB memory) via Bluetooth for behavior classification and odor detection. Finally, the detected odor signals were sent to the mobile robot, and the robot searched for the odor using an arbitrary searching algorithm.

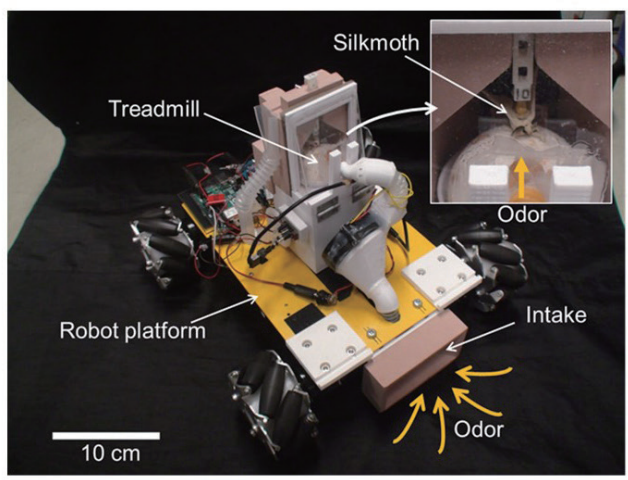

(a)

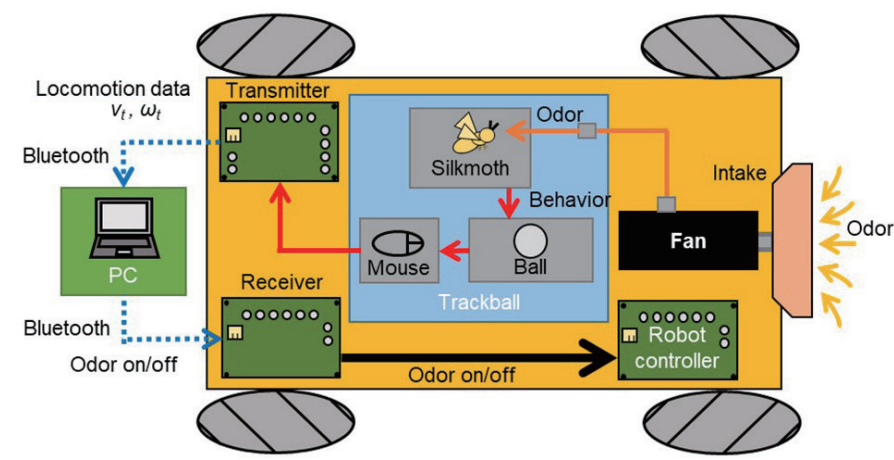

(b)

Fig. 2. (Color online) Odor-searching mobile robot with the insect-behavior-based olfactory sensor. (a) Appearance of the robot. (b) Diagram of the robot. 


\section{Behavior-based Olfactory Sensor}

\subsection{Experimental setup}

We used male adult silkmoths. Pupae were purchased from the National BioResource Project (https://shigen.nig.ac.jp/silkwormbase/), aged within ten days after eclosion, and maintained at $25{ }^{\circ} \mathrm{C}$. A male silkmoth was attached to a 3D-printed bar with a flexible attachment using an adhesive (G17, Konishi, Osaka, Japan). The animal preparation for the spherical treadmill was carried out by following a previous study. ${ }^{(5)}$ We collected behavioral data and the corresponding timing of an olfactory stimulus as teacher data to establish the classifier of silkmoth behaviors (24 data from 12 moths). Data were collected using the spherical treadmill on the robot [Fig. 3(a)]. The tethered silkmoth was stimulated by the major component of the conspecific female sex pheromone [bombykol: (E,Z)-10,12-hexadecadien-1-ol]. We applied $1000 \mathrm{ng}$ of bombykol dissolved in n-hexane to a piece of filter paper, placed the piece in a glass tube, connected the glass tube to an external olfactory stimulator, and released the odor in front of the air intake of the robot. The odor was delivered to the silkmoth through the onboard odor delivery system. In the previous study, we collected behavioral data on the basis of a constant stimulus condition (the stimulus was applied for $800 \mathrm{~ms}$, followed by a pause of $4200 \mathrm{~ms}$, then this procedure was repeated). ${ }^{(36)}$ However, the data should be collected on the basis of a stimulus condition close to an actual environment, where an odor arrives intermittently with variable durations (odor presence) and intervals (odor absence). ${ }^{(38)}$ To reproduce the realistic odor stimulation, we measured the temporal change in the odor concentration of the arena, where the odor searching experiment was conducted (for the conditions, see Sect. 4.2) with a photoionization detector (miniPID 200B, Aurora Scientific, Aurora, Canada), and determined the stimulus condition of

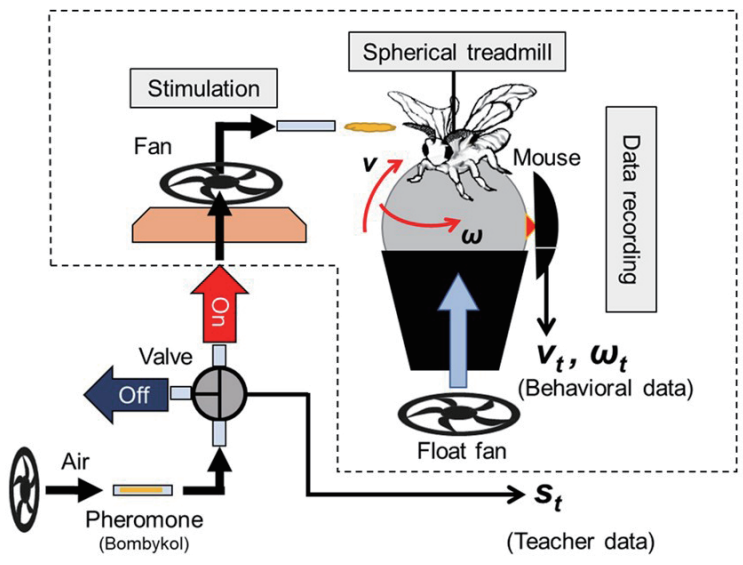

(a)
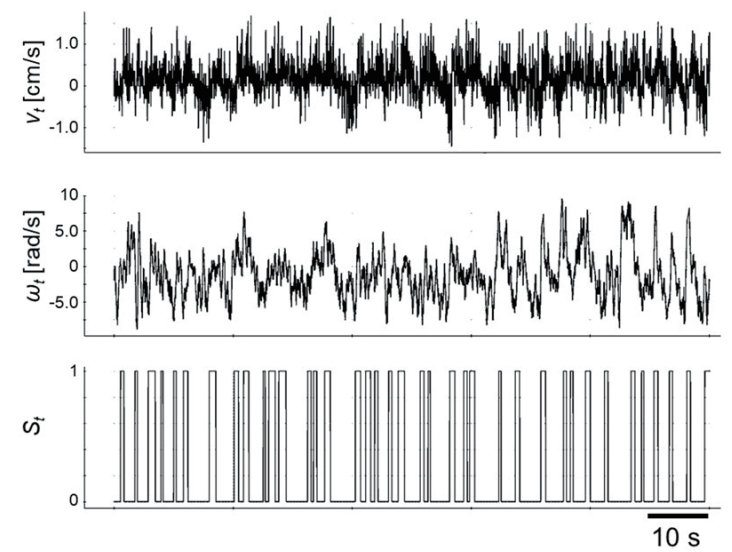

(b)

Fig. 3. (Color online) Experimental condition for locomotion data collection. (a) Behavioral experiment setup. The devices surrounded by the dashed line are attached to the robot. (b) Example of locomotion data $\left(v_{t}, \omega_{t}\right)$ and the timing of odor stimuli $\left(S_{t}\right)$. 
odor on the basis of the measurement. Odor stimuli were applied intermittently with variable durations $\left[t_{d}(\mathrm{~ms})\right]$ and intervals $\left[t_{i}(\mathrm{~ms})\right]$. The distributions of $t_{d}$ and $t_{i}\left(f_{1}\left(t_{s}\right), f_{2}\left(t_{i}\right)\right)$ were defined as

$$
\begin{gathered}
f_{1}\left(t_{d}\right)=\frac{1}{\mu} e^{-\frac{t_{d}}{\mu}}, \\
\left(300 \leq t_{d} \leq 1200 ; \mu=600\right), \\
f_{2}\left(t_{i}\right)=-\frac{2}{\left(R_{\text {max }}-R_{\min }\right)^{2}}\left(t_{i}-R_{\max }\right), \\
\left(R_{\text {min }} \leq t_{i} \leq R_{\text {max }} ; R_{\text {min }}=300, R_{\text {max }}=5000\right),
\end{gathered}
$$

where $\mu=600 \mathrm{~ms}$ is the mean of $t_{d}$, and $R_{\min }$ and $R_{\max }(\mathrm{ms})$ represent the lower and upper limits of $t_{i}$, respectively. A sample measurement of behavioral data $\left(v_{t}, \omega_{t}\right)$ in response to pheromone stimuli is shown in Fig. 3(b). The pheromone stimuli lasted for $100 \mathrm{~s}$ for each individual, and locomotion data were obtained.

\subsection{SVM classifier}

Figure 4 shows the overview of the SVM classifier. We trained a binary classifier (odor presence/absence) using the data collected over $100 \mathrm{~s}$. An SVM, which is a supervised machine learning model that solves classification problems, was used for this study because of its low computational cost. The radial basis function (RBF) kernel was applied to the classifier because it was reported to be better than other kernels in previous research in terms of estimating the timing of odor reception from silkmoths. ${ }^{(35)}$ The behavioral data $\left(v_{t}, \omega_{t}\right)$ were smoothed using the moving average for data processing. Then, six features were extracted as feature vectors, $\boldsymbol{x}_{t}$, at each time $t$,

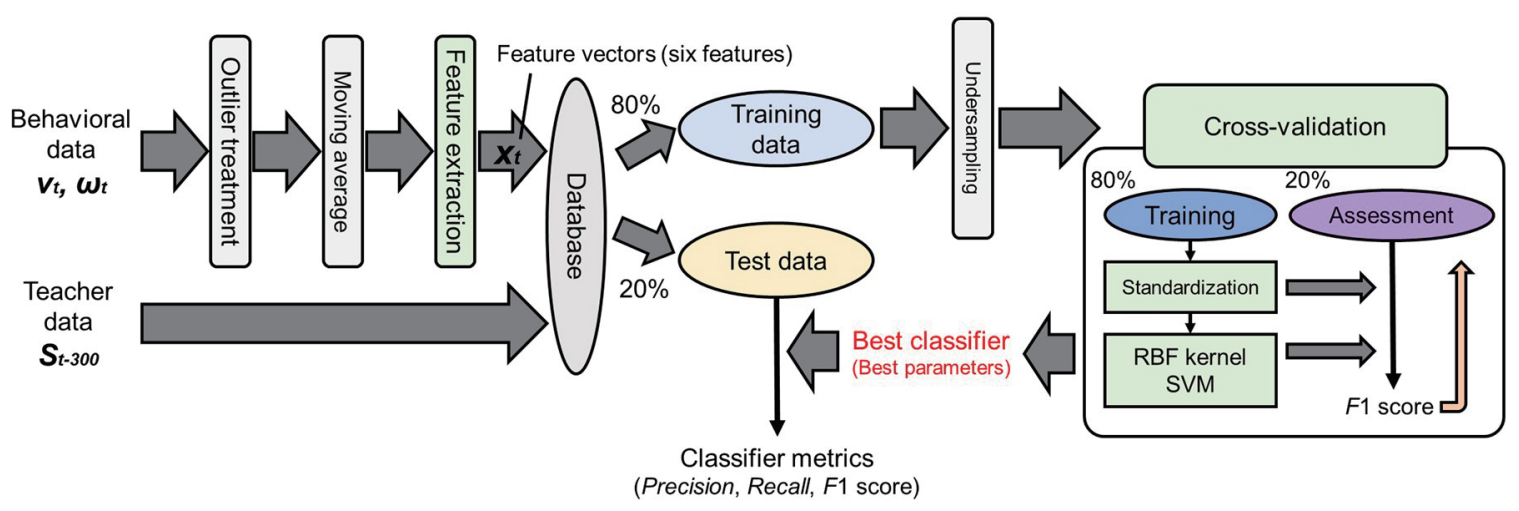

Fig. 4. (Color online) Overview of the classifier establishment. 


$$
\boldsymbol{x}_{t}=\left(\begin{array}{c}
v_{t} \\
\omega_{t} \\
v_{t-200} \\
\omega_{t-200} \\
v_{t-400} \\
\omega_{t-400}
\end{array}\right),
$$

where $v_{t}$ and $\omega_{t}$ represent the translational and angular velocities of the silkmoth after smoothing at time $t(\mathrm{~ms})$, respectively. The window width of the moving average $(300 \mathrm{~ms})$, the number of features (three each for $v_{t}$ and $\omega_{t}$ ), and time width for extracting features $(200 \mathrm{~ms}$ ) were determined on the basis of the performance of the classifier assessed by $F 1$ score (explained below). The timing of the olfactory stimulus (input), which was defined $300 \mathrm{~ms}$ after the electromagnetic valve was switched on/off owing to the time required for odor delivery, was used as teacher signals, $s_{t-300}$. The first $80 \mathrm{~s}$ of $\boldsymbol{x}_{t}$ and $s_{t-300}$ were used as training data, and the remaining $20 \mathrm{~s}$ were used as test data. The numbers of positive and negative data (responses to the presence and absence of the odor, respectively) for training were balanced by undersampling either. Then, we conducted cross-validation to obtain the parameters of the SVM with the RBF kernel. In the validation, the training data were divided into five parts, standardized, and input to the SVM. Finally, we determined the parameters of the classifier based on F1 score. The classifier was implemented using scikit-learn in Python. ${ }^{(39)}$ Combined with the classifier, the silkmoth and spherical treadmill worked as a binary olfactory sensor that predicted the presence of the odor. The time required to collect the data and establish the classifier was $5 \mathrm{~min}$.

\subsection{Evaluation of the behavior-based olfactory sensor}

The classifier was trained using individual test data and we calculated Precision, Recall, Specificity, and F1 score [Fig. 5(a) and Table 1]. Precision represents the ratio between correct

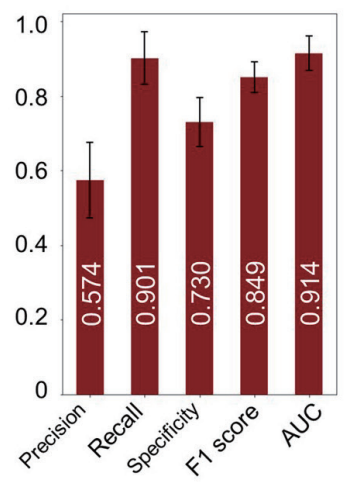

(a)

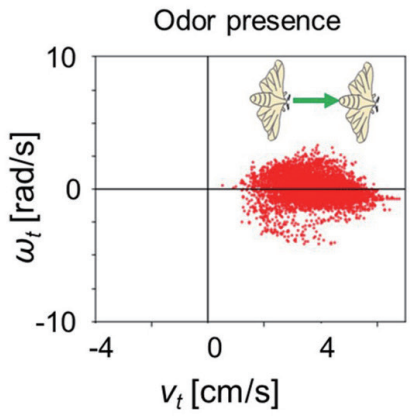

(b)

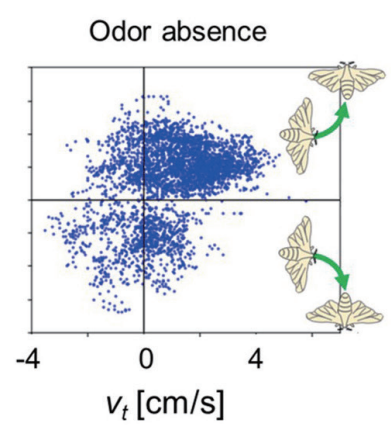

Fig. 5. (Color online) Classification results. (a) Classification metrics. Mean values (24 classifiers) and standard deviations are shown. (b) Distributions of $v_{t}$ and $\omega_{t}$ sorted by the classification criteria. 
Table 1

Results of odor searching test for all trials and the classifier performance.

\begin{tabular}{|c|c|c|c|c|c|c|c|c|c|}
\hline \multirow{2}{*}{ Algorithm } & \multirow[b]{2}{*}{ Moth } & \multicolumn{3}{|c|}{ Localization test } & \multicolumn{5}{|c|}{ Classification metrics } \\
\hline & & Localization & Time (s) & Distance (m) & Precision & Recall & Specificity & F1 score & AUC \\
\hline Spiral & 0 & Success & 128 & 11.8 & 0.650 & 0.928 & 0.780 & 0.857 & 0.963 \\
\hline Spiral & 1 & Success & 119 & 17.2 & 0.529 & 0.945 & 0.741 & 0.840 & 0.951 \\
\hline Spiral & 2 & Success & 204 & 33.4 & 0.621 & 0.796 & 0.752 & 0.879 & 0.865 \\
\hline Spiral & 3 & Success & 183 & 16.4 & 0.510 & 0.982 & 0.717 & 0.867 & 0.912 \\
\hline Spiral & 4 & Failure & - & - & 0.524 & 0.695 & 0.803 & 0.848 & 0.878 \\
\hline Spiral & 5 & Success & 113 & 8.9 & 0.556 & 0.869 & 0.630 & 0.826 & 0.841 \\
\hline Spiral & 6 & Success & 202 & 16.6 & 0.701 & 0.953 & 0.746 & 0.901 & 0.962 \\
\hline Spiral & 7 & Success & 107 & 10.2 & 0.505 & 1.00 & 0.712 & 0.895 & 0.949 \\
\hline Spiral & 8 & Success & 87 & 4.1 & 0.480 & 0.918 & 0.753 & 0.865 & 0.903 \\
\hline Spiral & 9 & Success & 196 & 13 & 0.628 & 0.902 & 0.697 & 0.888 & 0.916 \\
\hline Spiral & 10 & Success & 204 & 25.5 & 0.818 & 0.930 & 0.612 & 0.888 & 0.957 \\
\hline Spiral & 11 & Success & 55 & 3.1 & 0.619 & 0.942 & 0.749 & 0.840 & 0.949 \\
\hline Hex-path & 0 & Success & 76 & 6.2 & 0.611 & 0.88 & 0.643 & 0.851 & 0.899 \\
\hline Hex-path & 1 & Success & 60 & 4.8 & 0.562 & 0.961 & 0.689 & 0.807 & 0.930 \\
\hline Hex-path & 2 & Failure & - & - & 0.675 & 0.898 & 0.696 & 0.855 & 0.935 \\
\hline Hex-path & 3 & Failure & - & - & 0.627 & 0.824 & 0.852 & 0.882 & 0.923 \\
\hline Hex-path & 4 & Success & 63 & 4.9 & 0.502 & 1.00 & 0.712 & 0.865 & 0.934 \\
\hline Hex-path & 5 & Success & 38 & 3.1 & 0.567 & 0.959 & 0.742 & 0.846 & 0.962 \\
\hline Hex-path & 6 & Success & 103 & 8.2 & 0.562 & 0.839 & 0.829 & 0.880 & 0.945 \\
\hline Hex-path & 7 & Failure & - & - & 0.717 & 0.850 & 0.760 & 0.828 & 0.913 \\
\hline Hex-path & 8 & Failure & - & - & 0.519 & 0.934 & 0.726 & 0.856 & 0.919 \\
\hline Hex-path & 9 & Failure & - & - & 0.463 & 0.901 & 0.834 & 0.788 & 0.933 \\
\hline Hex-path & 10 & Failure & - & - & 0.322 & 0.872 & 0.755 & 0.824 & 0.811 \\
\hline Hex-path & 11 & Success & 37 & 4.2 & 0.500 & 0.840 & 0.602 & 0.706 & 0.795 \\
\hline
\end{tabular}

Spiral: spiral surge algorithm

predictions and all positive predictions. Recall represents the true positive rate, which is the ratio between correct positive predictions and all actual positives. Specificity represents the true negative rate, which is the ratio between correct negative predictions and all actual negatives. $F 1$ score represents the harmonic mean of Precision and Recall, which becomes high only when Precision and Recall are high. These classification metrics were calculated using the classifier and test data;

$$
\begin{gathered}
\text { Precision }=\frac{T P}{T P+F P}, \\
\text { Recall }=\frac{T P}{T P+F N}, \\
\text { Specificity }=\frac{T N}{T N+F P}, \\
F 1 \text { score }=\frac{2 \times \text { Precision } \times \text { Recall }}{\text { Precision }+ \text { Recall }},
\end{gathered}
$$


where $T P, F P, T N$, and $F N$ are the absolute numbers of true positives, false positives, true negatives, and false negatives, respectively.

The mean and standard deviation of the classification metrics were as follows: Precision $=0.574 \pm 0.101$, Recall $=0.901 \pm 0.070$, Specificity $=0.731 \pm 0.066$, and $F 1$ score $=0.849 \pm 0.041$ (Table 1). Recall and F1 score were comparable to the values reported in the previous studies of behavior classification in silkmoths (Recall, $0.72-0.93$; $^{(34)} F 1$ score, $\left.0.71-0.91^{(35)}\right)$. However, Precision was lower than the value reported in a previous study $\left(0.60-0.88^{(34)}\right)$. Figure 5(b) shows the distributions of $v_{t}$ and $\omega_{t}$ obtained from a single trial when the classifier predicted the presence or absence of the odor. The absolute values of $\omega_{t}$ and $v_{t}$ were high and low, respectively, when the classifier predicted odor absence, which indicated the zigzagging turns of the silkmoth. In contrast, the absolute values of $\omega_{t}$ and $v_{t}$ were low and high, respectively, when the classifier predicted odor presence, which indicated a surge. Figure 6 shows the performance characteristics of the seven representative classifiers represented as receiver operating characteristic (ROC) curves and their predicted timings of odor presence/ absence compared with the actual timings. The area under an ROC curve (AUC) enables us to evaluate Recall and Specificity. $\mathrm{AUC}=1$ indicates a perfect classifier and $\mathrm{AUC}=0.5$ indicates a random classifier. The comparison of the predicted and actual timings showed that their similarity was closely related to the AUC.
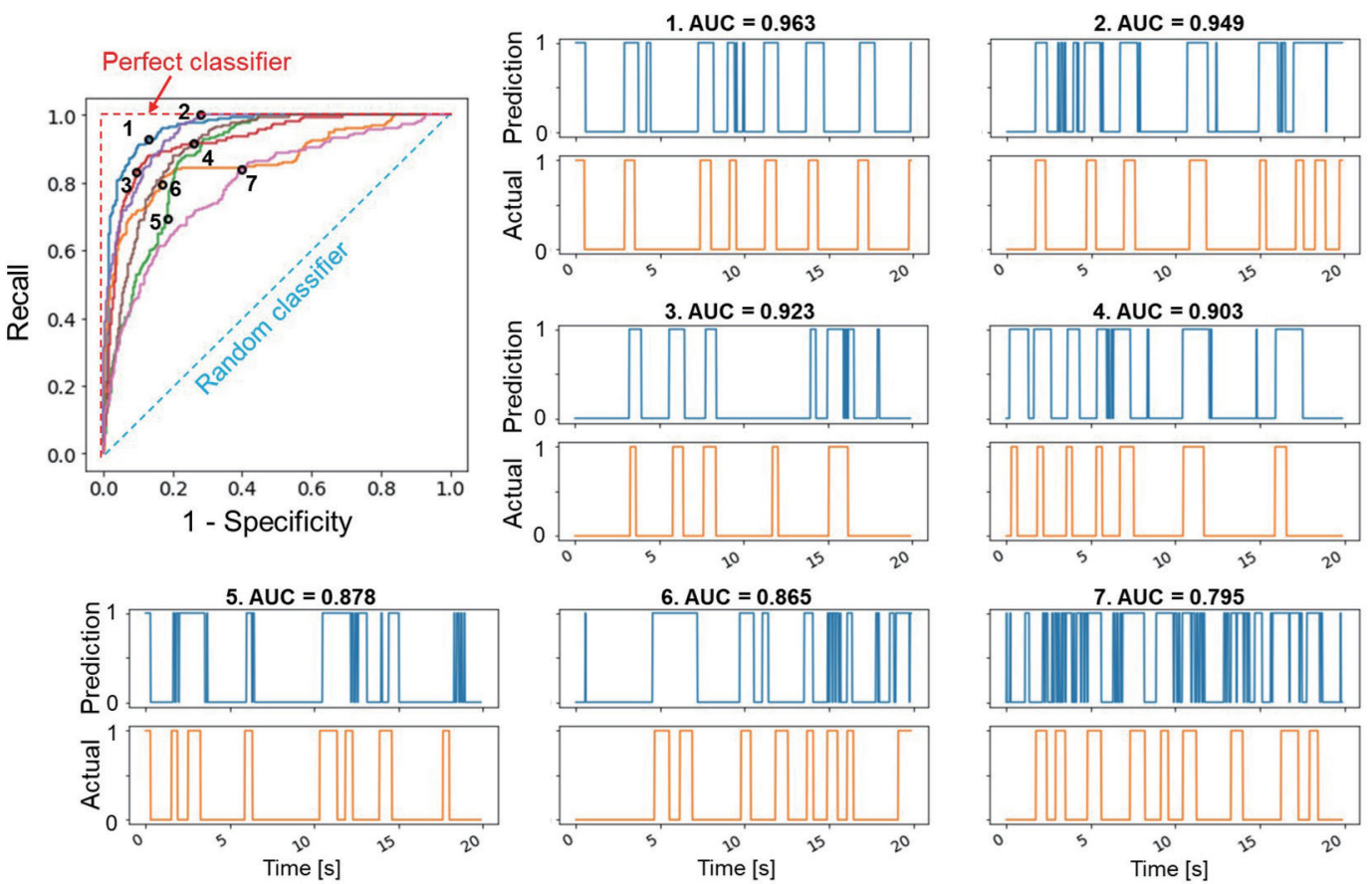

Fig. 6. (Color online) ROC curves of seven representative classifiers (including the highest and lowest AUCs, see Table 1) and corresponding prediction of odor presence/absence. The black point in each ROC curve indicates the threshold (0) decision value used for the binary classification. In each classification result, the predicted and actual odor presence (1) or absence (0) values are shown in the upper and lower traces, respectively. 


\section{Robot Experiment}

\subsection{Animal and robot preparation}

The robot experiment was conducted after collecting the behavioral data and establishing the classifier. The treadmill was surrounded by white paper to minimize the effect of self-induced optic flow because male silkmoths respond to optic flow and turn during a surge. ${ }^{(40)}$ An acrylic cover with white balls was attached to the top of the robot to track the position of the robot using software (DIPP Motion V, Ditect, Tokyo, Japan).

\subsection{Experimental conditions}

The robot experiment was conducted in an area [2.5 m (W) $\times 3 \mathrm{~m}(\mathrm{~L})$, Fig. 7(a)] in a room. The odor source was a piece of filter paper on which $5 \mu \mathrm{g}$ of bombykol was applied. The source was set at the end of the area, and a fan blew air from behind the source (the windspeed was 1-2 $\mathrm{m} / \mathrm{s}$ at $1 \mathrm{~m}$ downwind from the source). A preliminary observation showed that six out of seven silkmoths walked toward the odor source ( $1 \mu \mathrm{g}$ bombykol) from a distance of 3-4 m; thus, the 5 $\mu \mathrm{g}$ of bombykol was sufficient for the silkmoths to exhibit a surge in response to pheromone reception from a distance of 3-4 m. The position of the source was defined as the origin. The robot started searching for the odor source from an area $(-50 \mathrm{~cm}<x<50 \mathrm{~cm}$, $-300 \mathrm{~cm}<y<-250 \mathrm{~cm})$, initially heading in the direction of $\pm 45^{\circ}\left(0^{\circ}\right.$ indicates the orientation of the $y$-axis). The experiment was continued for $5 \mathrm{~min}$, and the robot movements were captured by a video camera. The robot was assumed to have reached the odor source at a distance of $50 \mathrm{~cm}$ from the source. The trial was assumed to have failed if the robot did not reach the source within $5 \mathrm{~min}$ or exited the area. Robot experiments were also conducted without the odor source as control experiments.

We characterized the odor plume structure before performing the robot experiments with the behavior-based odor sensor. A paper containing $3 \mathrm{~mL}$ of $70 \%$ ethanol was placed as an odor

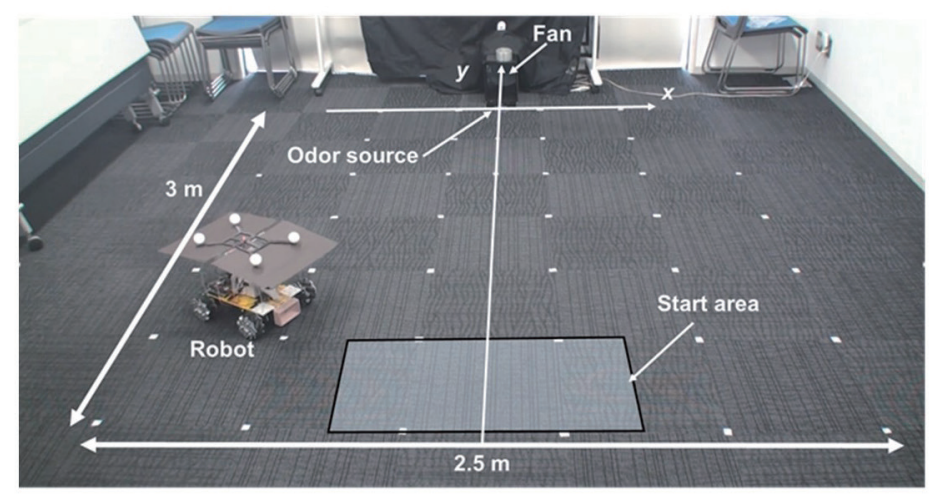

(a)

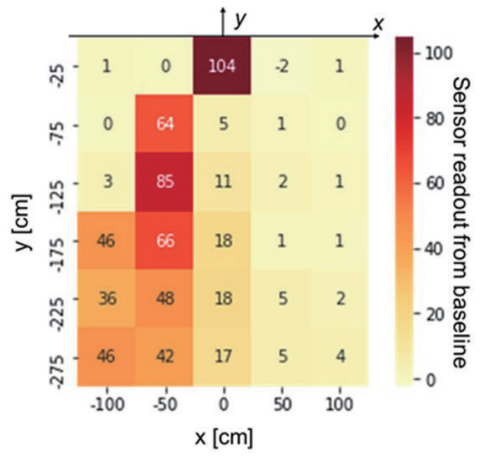

(b)

Fig. 7. (Color online) Experimental conditions. (a) Experimental area for odor searching experiments. (b) Distribution of odor concentration in the area. 
source, and the odor was blown by a fan. The odor concentration was measured at $50 \mathrm{~cm}$ intervals using a semiconductor gas sensor (MiCS-5524, SGX Sensortech, Neuchatel, Switzerland) via an Arduino Uno. The results indicated that the distribution of the odor plume was shifted by approximately $-50 \mathrm{~cm}$ from the midline [Fig. 7(b), $y$-axis].

\subsection{Searching algorithm}

The robot was designed to track an odor plume based on an arbitrary searching algorithm triggered by the presence of the odor estimated by a classifier [Fig. 1(b)]. We employed two reactive algorithms, the spiral surge ${ }^{(41)}$ and hex-path ${ }^{(42)}$ algorithms, owing to their simplicity of implementation in the robot (Fig. 8) among the various odor searching algorithms proposed previously. ${ }^{(4)}$ The spiral surge algorithm is for a turbulent odor flow, whereas the hex-path algorithm is used to search for an odor under the ground, where diffusion is the dominant form of odor distribution.

In the spiral surge algorithm, a surge occurs when there is an odor (moving straight) and a spiral occurs when there is no odor (following an Archimedean spiral). In this study, the forward velocity during a surge was set as $10 \mathrm{~cm} / \mathrm{s}$. The forward velocity $v_{s}(t)$ and angular velocity $\omega_{s}(t)$ during a spiral were defined as

$$
v_{s}(t)=\frac{S P I R A L_{-} \text {WIDTH }}{\pi / 2} \omega_{s}(t) \int_{0}^{t} \omega_{S}(u) d u
$$
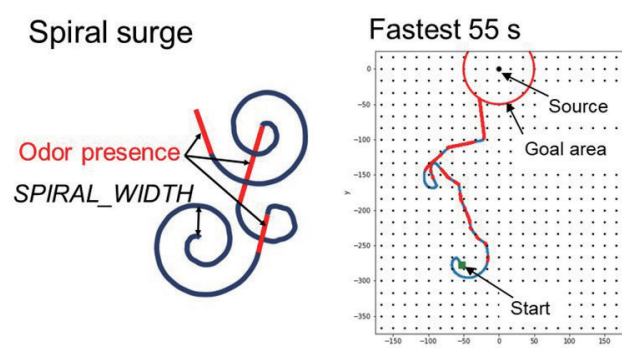

Hex-path
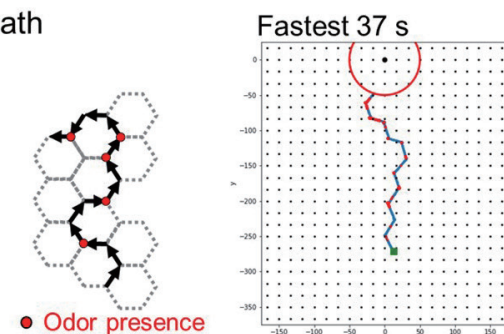

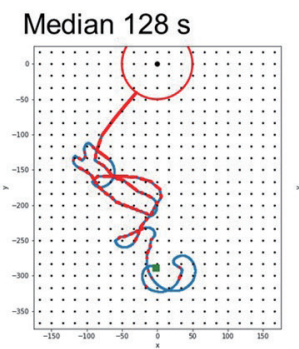

(a)

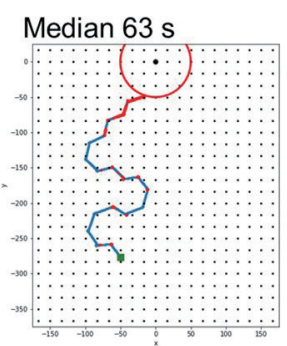

(b)
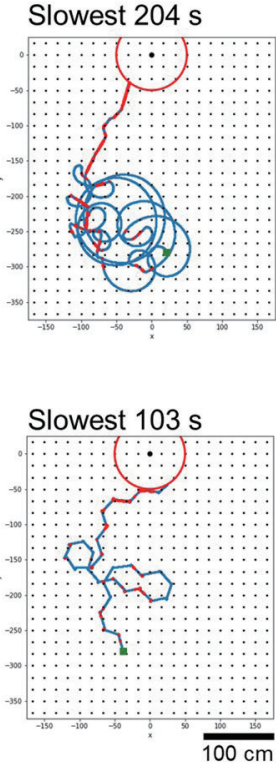

Fig. 8. (Color online) Trajectories of the robot implemented with the (a) spiral surge and (b) hex-path algorithms. The fastest, median, and slowest trials among successful trials are shown. The segmented trajectories shown in red and blue indicate when the sensor predicts the presence and absence of the odor, respectively. 


$$
\omega_{S}(t)=\max (0.3,0.8-0.05 t),
$$

where SPIRAL_WIDTH is the spiral width, which was fixed at $50 \mathrm{~cm}$. If the robot did not detect an odor more than $20 \mathrm{~s}$ after the previous detection, then $v_{s}(t), \omega_{s}(t)$, and the gyration radius were not updated and remained constant. The direction of the spiral was determined randomly.

In the hex-path algorithm, a robot moves along the outline of a single hexagon in the same direction (clockwise or counterclockwise) if the odor concentration decreases and switches the direction of rotation at the vertices if the concentration increases. As the output of the behaviorbased odor sensor was binary, the frequency of odor detection during the forward movement on each side of a hexagon was used instead of the increase/decrease in odor concentration. The forward velocity, the length of a single side, and the angular velocity at a vertex were set as $18 \mathrm{~cm} / \mathrm{s}, 20 \mathrm{~cm}$, and $1.0 \mathrm{rad} / \mathrm{s}$, respectively.

Before performing the robot experiment with the behavior-based odor sensor, we conducted odor searching experiments by employing the robot with a gas sensor in an ethanol plume. The robot in which these algorithms were implemented localized the odor source in all trials (five trials for each algorithm, data not shown).

\subsection{Results}

The results of odor source localization for all trials and the corresponding classification metrics are shown in Table 1. There were no significant differences between the classification metrics for the spiral surge and hex-path algorithms, except for $F 1$ score (spiral surge, $0.866 \pm 0.024$; hex-path, $0.832 \pm 0.049 ; P<0.05$, $t$-test). Figure 8 shows the fastest, median, and slowest trajectories in which the robot localized the odor source. The robot moved in a wide range when it was far from the odor source. The robot finally reached the odor source by following a similar path in both algorithms, i.e., it moved in a straight line from the left of the odor source. The robot with the spiral surge algorithm localized the odor source in 11 out of 12 trials (success rate, 92\%), whereas the robot with the hex-path algorithm localized it in 6 out of 12 trials $(50 \%)$. The robot moved out of the area in all failed trials. The time and travel distance required for localization for the spiral surge algorithm were longer than those for the hex-path algorithm [spiral surge, $128 \mathrm{~s}(110,199)$ and $13.0 \mathrm{~m}(9.6,16.9)$; hex-path, $62 \mathrm{~s}(38,76)$ and $4.9 \mathrm{~m}$ $(4.2,6.2)$; median (interquartile range)], and there were significant differences between the values (time, $P<0.01$; travel distance, $P<0.05$; Mann-Whitney $U$ test at a significance level of $P<0.05)$.

We compared the classification metrics between successful and failed trials for the hex-path algorithm (Fig. 9; we did not compare these metrics for the spiral surge algorithm because only one trial failed) to investigate the relationship between the performance of the classifier and the success rate of localization. Although there were no significant differences between the metrics for successful and failed trials $(P>0.05, t$-test $), R e c a l l$ and Specificity showed different tendencies. Recall for successful trials was higher than that for failed trials (successful, $0.913 \pm 0.69$; failed, $0.880 \pm 0.039$ ), whereas Specificity for successful trials was lower than that 


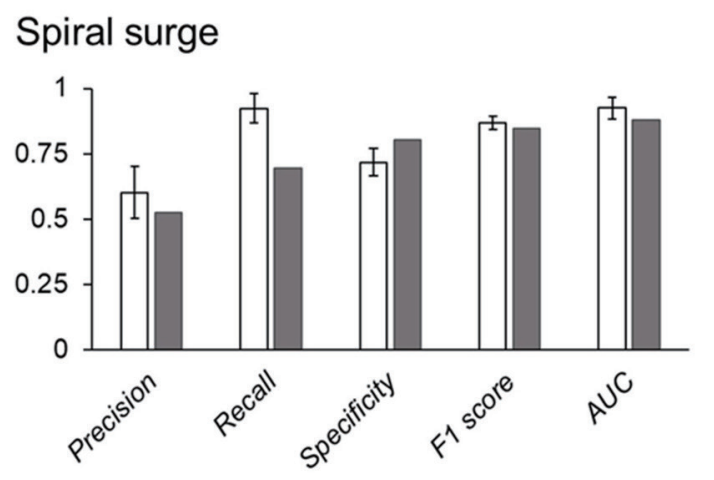

(a)

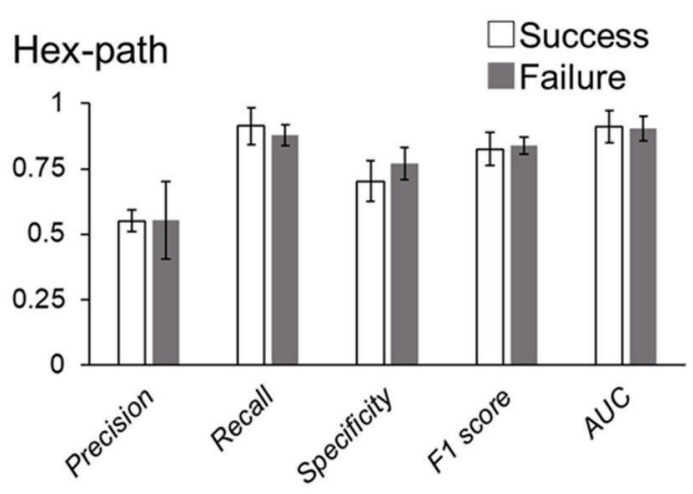

(b)

Fig. 9. Comparison of classification metrics between successful and failed trials of the (a) spiral surge and (b) hexpath algorithms. The means and standard deviations are shown.

for failed trials (successful, $0.703 \pm 0.079$; failed, $0.771 \pm 0.061$ ). A similar tendency was observed in Recall and Specificity for the spiral surge algorithm, even though only one trial failed in this case.

The odor detection probability in each $50 / 3 \times 50 / 3 \mathrm{~cm}^{2}$ mesh was calculated to investigate whether the behavior-based odor sensor captured the plume structure [Figs. 10(a) and 10(b)]. The odor detection probability was calculated by dividing the number of odor detections by the number of predictions in each mesh. The detection probability is 1 if the classifier always predicts odor presence in a mesh and 0 if the classifier always predicts odor absence. The detection probability was high on the leeward left side of the odor source for all successful and failed trials for the two algorithms. The sensor rarely predicted odor presence when the odor source was removed [no odor, Figs. 10(a) and 10(b)]. The averaged detection probability for all trials $[N=24$, Fig. 10(c)] showed that the plume structure shifted to the left; this result was similar to that obtained using the gas sensor [Fig. 7(b)]. The arrows in Fig. 10 indicate the mean vector of the robot's direction in each mesh. A long arrow indicates that the robot is oriented in similar directions between trials. The arrows became longer and directed toward the odor source as the detection probability increased in successful trials. In a few meshes, there were long arrows and high detection probabilities away from the source. However, it is uncertain whether the high detection probabilities in these meshes are reproducible because the robot passed through these meshes in only one or two trials as shown in Fig. 10(d).

\section{Discussion}

We developed an olfactory sensor based on the behavior of silkmoths and implemented it in a mobile robot for odor searching. The robot with the sensor successfully localized an odor source using artificial searching algorithms. Several previous studies have used the behaviors and flight muscle activities of silkmoths to classify behaviors and estimate odor acceptance using machine 


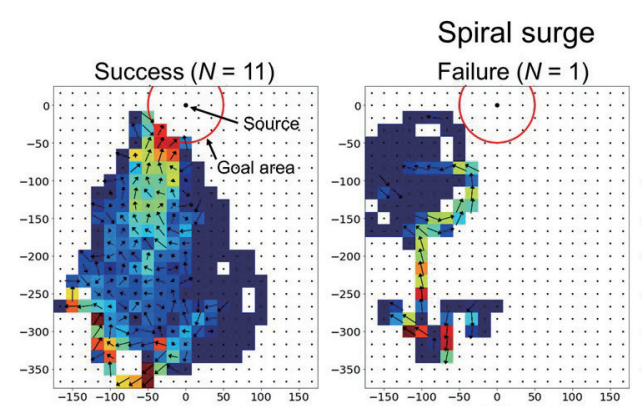

(a)
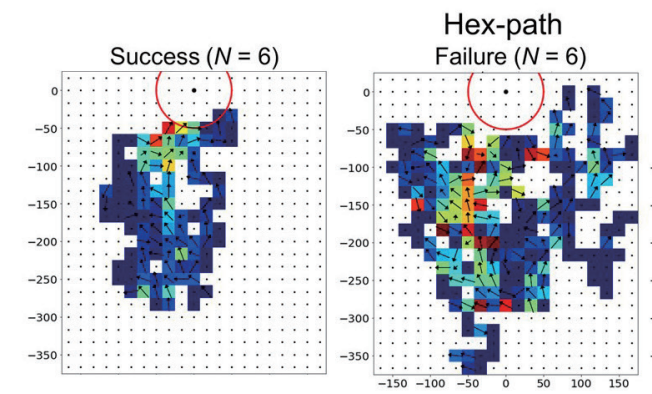

(b)
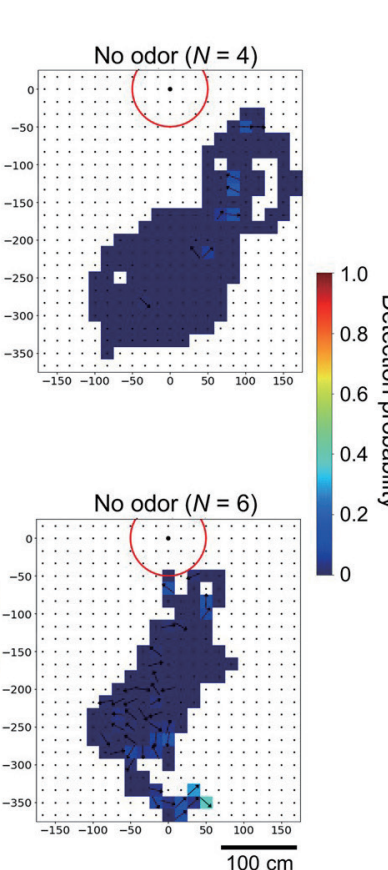

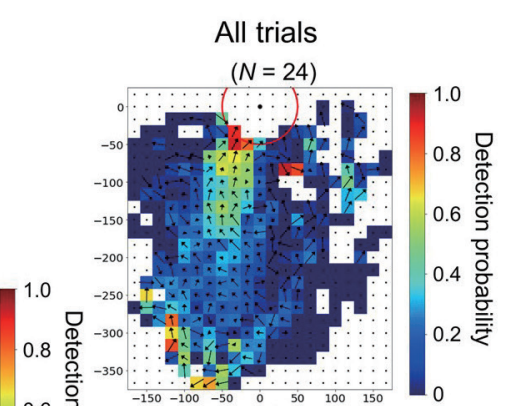

(c)

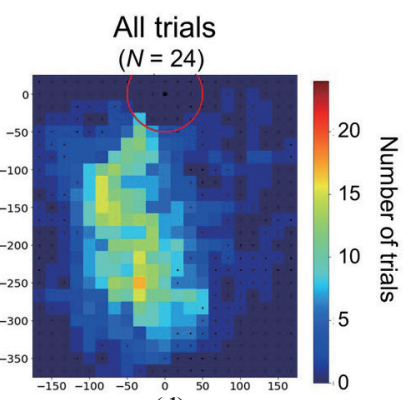

(d)

Fig. 10. (Color online) Probability map of odor detection. $(a, b)$ Detection probability in successful trials, failed trials, and trials without the odor plume for the (a) spiral surge and (b) hex-path algorithms. The meshes without color are those that the robot does not pass through. The direction and length of the arrows indicate the mean vector of the robot direction in each mesh. (c) Detection probability for all trials under the odor plume (24 trials). (d) Distribution of the number of trials in which the robot passes through each mesh (max: 24 trials).

learning. ${ }^{(34-36)}$ High classifier performance is preferable; however, it is not clear whether the performance achieved in previous studies is sufficient for the odor searching task. In this study, the classifier performance was characterized by classification metrics. Even though the classifier performance did not exceed that obtained in previous studies, it was sufficient for the mobile robot to locate the odor source. Further improvement of classifiers by adding other behavioral parameters such as wingbeat patterns and body and antennal movements ${ }^{(33)}$ will increase the classification performance to close to that of silkmoths. The direct use of insect odor sensing has three advantages: high sensitivity, fast response, and high selectivity. Recent studies have shown that some types of semiconductor gas sensors exhibit fast responses comparable to insect EAGs $(2-5 \mathrm{~Hz}) .{ }^{(43,44)}$ Furthermore, a mobile robot equipped with localized surface plasmon resonance gas sensors resolved the temporal fluctuation of chemical signals at more than $25 \mathrm{~Hz}$ and tracked odors. ${ }^{(45)}$ Therefore, the advantage of high response speed in insect olfaction may be diminished relative to the response speed of recent artificial chemical sensors. On the other hand, high sensitivity and high selectivity are still advantages of insect-based biosensors. The EAG-based mobile robot was the first such mobile robot to be developed. ${ }^{(21)}$ More recently, a mobile robot equipped with a chemical sensor based on a mosquito's olfactory receptors has been reported. ${ }^{(46)}$ Our behavior-based sensor is a new direction in the use of insect olfaction for mobile robots. Here, we discuss the features of our system, the relationship between the classification metrics 
and performance of odor source localization, and the perspective of odor detection devices based on insect behavior.

The difference between individuals is a major issue in sensors that use biological systems. ${ }^{(29,30)}$ In the case of behavior classification using machine learning, there is an evident difference between the classifier performance characteristics among individuals. ${ }^{(34-36)}$ Therefore, the establishment of the classifier by individuals before the odor searching experiment is preferable, and it is necessary to simplify the establishment procedure for practical use for odor searching. In our system, the same robot platform was used to establish the classifier and perform the odor searching experiment. After a silkmoth was tethered and placed on the spherical treadmill, only 5 min was required to acquire the training data and establish a classifier for each individual. Then, the robot performed the odor searching task. Therefore, our system and the procedure used to establish a classifier increase the practicality of behavior-based olfactory sensors.

The previous studies that used insect behavior as an olfactory sensor were only for chemical detection. ${ }^{(27,29-31,47)}$ We demonstrated that behavior-based odor detection can be combined with robotic odor searching using arbitrary searching algorithms. The algorithms employed in this study were reactive strategies. The robots implemented in reactive strategies need to respond to an instantaneous odor input and execute preprogrammed behavior patterns. Therefore, the olfactory sensors require high response speed with short latency. The sampling frequency of 50 $\mathrm{Hz}$ used for obtaining locomotion data was sufficient to capture the silkmoth behavior. ${ }^{(43)}$ The estimated time delay, excluding the time required for sensory-motor processing in the silkmoths, was $1 \mathrm{~s}$, i.e., $300 \mathrm{~ms}$ for odor delivery, $300 \mathrm{~ms}$ for the window width of the moving average, and $400 \mathrm{~ms}$ for the feature extraction [see Eq. (3)]. The time delay of $1 \mathrm{~s}$ might be problematic because the studies on silkmoth-driven odor-searching robots have revealed that the odor searching performance decreases as the time delay becomes longer than $400 \mathrm{~ms} .{ }^{(48)}$ However, this occurs if the searching algorithm of actual silkmoths is employed. In contrast to the silkmoth-driven robot, the robot in this study is driven by arbitrary searching algorithms and the parameters can be modified. Therefore, it is possible to optimize the parameters such as the translational and angular velocities of the algorithms to maximize the odor searching performance even with a time delay of $1 \mathrm{~s}$. Furthermore, we can select the algorithms on the basis of odor environments. This study used the spiral surge and hex-path searching algorithms. Different success rates were obtained using the two algorithms (92\% for spiral surge and 50\% for hex-path). However, the difference was acceptable considering the turbulent odor environment in the area. If the robot with the hex-path algorithm loses the odor plume, it becomes difficult for the robot to re-enter the plume because the search area is hexagonal in the absence of the odor. In contrast, if the robot searches within the plume and continuously detects the odor, e.g., when diffusion is dominant, a linear path can be selected to rapidly locate the odor source, such as in the case of the fastest trial shown in Fig. 8(b).

The classifier performance obtained in this study was sufficient for performing odor searching using the spiral surge algorithm, although Precision was not particularly high (0.574). The low Precision was due to the relatively high false-positive rate, i.e., silkmoths performed a surge in the absence of the odor. One of the reasons for this could be a prolonged surge after odor 
reception, which has been reported to be advantageous for insects performing an upwind surge outside a plume. ${ }^{(49,50)}$ The high success rate of the spiral surge algorithm irrespective of the relatively low Precision is related to the odor environment. The robot frequently spiraled away from the odor source. In this case, a false positive leads to a spontaneous surge and shifts the center of the spiral. This expands the search area and may increase the probability of receiving odor. This does not mean that low Precision is better because such spontaneous factors can be implemented in the algorithm if a sensor with high Precision is employed. Therefore, the characteristics of the olfactory sensor and the search algorithm are complementary, and low classification metrics do not necessarily reduce odor searching performance.

We did not find significant differences between the classification metrics for successful and failed trials in the experiments with the hex-path algorithm, in which the success rate was $50 \%$. Therefore, the success rate might depend on whether or not the robot is continuously in the odor plume, rather than the difference between the classification metrics. There were relatively notable differences between Recall and Specificity, although the differences were not significant. The mean Recall of successful trials was higher than that of failed trials. This indicates that the success rate will increase if we select classifiers with higher Recall (all three trials with Recall above 0.95 were localized). In contrast, the mean Specificity of successful trials was lower than that of failed trials. This might be due to the same reason as that discussed above. In the future, the relationship between the classifier performance and the odor searching capability will be quantified by performing experiments using classifiers with different ranges of these metrics.

In this study, we used silkmoths and the robot searched only for the conspecific sex pheromone. Recently, an insect-based biorobot that can identify target chemicals based on multiple neural signals from the locust brain has been reported. ${ }^{(51)}$ Although silkmoths exhibit the searching behavior only for bombykol, recent progress in genetic tools has enabled the generation of transgenic silkmoths, which express other types of olfactory receptors on pheromone receptor cells. ${ }^{(52)}$ These silkmoths can innately respond to odors and localize odor sources as if they are receiving bombykol. The application of these silkmoths to our robot can enable the robot to detect a specified odor and localize a source.

\section{Conclusions}

We developed an odor-searching mobile robot with an insect-behavior-based olfactory sensor. An SVM-based behavior classifier predicted the presence and absence of the odor on the basis of the locomotor activity of an onboard silkmoth. Two artificial reactive searching algorithms were employed to drive the robot using the classifier output. The classifier performance was comparable to that of previous studies, and we found that the sensor performance was sufficient for the robot to locate an odor source using the conventional spiral surge algorithm. We also developed a procedure to quickly create a classifier for each individual on the mobile robot, which increased the practicality of the behavior-based sensor. Further development of genetic manipulation of olfactory receptors in silkmoths will increase the selectivity of the sensor and enhance the performance of searching for specific odors in a real environment. 


\section{Acknowledgments}

We thank Shigeru Matsuyama for providing purified bombykol. This work was supported by the joint research fund from NEC Corporation.

\section{References}

1 R. A. Russell: Int. J. Robot. Res. 20 (2001) 144. https://doi.org/10.1177/02783640122067318

2 G. Kowadlo and R. A. Russell: Int. J. Robot. Res. 27 (2008) 869. https://doi.org/10.1177/0278364908095118

3 H. Ishida, Y. Wada, and H. Matsukura: IEEE Sens. J. 12 (2012) 3163. https://doi.org/10.1109/jsen.2012.2208740

4 X.-X. Chen and J. Huang: Rob. Auton. Syst. 112 (2019) 123. https://doi.org/10.1016/j.robot.2018.11.014

5 N. Ando and R. Kanzaki: Arthropod Struct. Dev. 46 (2017) 723. https://doi.org/10.1016/j.asd.2017.02.003

6 V. A. Webster-Wood, O. Akkus, U. A. Gurkan, H. J. Chiel, and R. D. Quinn: Sci. Rob. 2 (2017). https://doi. org/10.1126/scirobotics.aap9281

7 M. Denny: Air and Water: The Biology and Physics of Life's Media (Princeton University Press, 1993).

8 J. D. Bohbot and S. Vernick: Biosens.-Basel 10 (2020). https://doi.org/10.3390/bios10030026

9 J. S. Elkinton, C. Schal, T. Onot, and R. T. Cardé: Physiol. Entomol. 12 (1987) 399. https://doi. org/10.1111/j.1365-3032.1987.tb00766.x

10 C. Wall and J. N. Perry: Entomol. Exp. Appl. 44 (1987) 5. https://doi.org/10.1111/j.1570-7458.1987.tb02232.x

11 T. Dekker and R. T. Carde: J. Exp. Biol. 214 (2011) 3480. https://doi.org/10.1242/jeb.055186

12 D. Schneider: Z. vergl. Physiol. 40 (1957) 8. https://doi.org/10.1007/bf00298148

13 J. Bau, K. A. Justus, C. Loudon, and R. T. Cardé: Chem. Senses 30 (2005) 771. https://doi.org/10.1093/chemse/ bji069

14 P. Szyszka, R. C. Gerkin, C. G. Galizia, and B. H. Smith: Proc. Natl. Acad. Sci. U. S. A. 111 (2014) 16925. https://doi.org/10.1073/pnas.1412051111

15 J. D. Bohbot and J. C. Dickens: Front. Cell. Neurosci. 6 (2012) 29. https://doi.org/10.3389/fncel.2012.00029

16 J. N. C. van der Pers and A. K. Minks: Entomol. Exp. Appl. 87 (1998) 209.

17 S. Schütz, M. J. Schöning, P. Schroth, Ü. Malkoc, B. Weißbecker, P. Kordos, H. Lüth, and H. E. Hummel: Sens. Actuators, B 65 (2000) 291. https://doi.org/10.1016/s0925-4005(99)00325-1

18 A. J. Myrick, K. C. Park, J. R. Hetling, and T. C. Baker: Bioinspir. Biomim. 3 (2008) 046006. https://doi. org/10.1088/1748-3182/3/4/046006

19 K. C. Park, S. A. Ochieng, J. Zhu, and T. C. Baker: Chem. Senses 27 (2002) 343. https://doi.org/10.1093/ chemse/27.4.343

20 A. J. Myrick, K. C. Park, J. R. Hetling, and T. C. Baker: J. Chem. Ecol. 35 (2009) 118. https://doi.org/10.1007/ $\underline{\text { s10886-008-9582-4 }}$

21 Y. Kuwana, I. Shimoyama, and H. Miura: Proc. 1995 IEEE/RSJ International Conf. Intelligent Robots and Systems: Human Robot Interaction and Cooperative Robots 2 (IEEE, 1995) 530-535. https://doi.org/10.1109/ IROS.1995.526267

22 D. Martinez, L. Arhidi, E. Demondion, J. B. Masson, and P. Lucas: J. Visualized Exp. 90 (2014) e51704. https:// doi.org/10.3791/51704

23 R. Kanzaki: Int. Congr. 1301 (2007) 7. https://doi.org/10.1016/j.ics.2006.12.037

24 M. J. Anderson, J. G. Sullivan, T. K. Horiuchi, S. B. Fuller, and T. L. Daniel: Bioinspir. Biomim. 16 (2020) 026002. https://doi.org/10.1088/1748-3190/abbd81

25 Y. Kuwana, S. Nagasawa, I. Shimoyama, and R. Kanzaki: Biosens. Bioelectron. 14 (1999) 195. https://doi. org/10.1016/s0956-5663(98)00106-7

26 B. Lan, R. Kanzaki, and N. Ando: Sensors 19 (2019). https://doi.org/10.3390/s19204574

27 G. C. Rains, J. K. Tomberlin, M. D'Alessandro, and W. J. Lewis: Trans. ASAE 47 (2004) 2145. https://doi. org/10.13031/2013.17785

28 M. Schott, C. Wehrenfennig, T. Gasch, and A. Vilcinskas: Adv. Biochem. Eng. Biotechnol. 136 (2013) 101. https://doi.org/10.1007/10_2013_210

29 G. C. Rains, J. K. Tomberlin, and D. Kulasiri: Trends Biotechnol. 26 (2008) 288. https://doi.org/10.1016/j. tibtech.2008.02.007

30 O. Leitch, A. Anderson, K. P. Kirkbride, and C. Lennard: Forensic Sci. Int. 232 (2013) 92. https://doi. org/10.1016/j.forsciint.2013.07.004

31 G. C. Rains, S. L. Utley, and W. J. Lewis: Biotechnol. Progr. 22 (2006) 2. https://doi.org/10.1021/bp050164p 
32 T. L. King, F. M. Horine, K. C. Daly, and B. H. Smith: IEEE Trans. Instrum. Meas. 53 (2004) 1113. https://doi. org/10.1109/tim.2004.831455

33 R. Kanzaki, N. Sugi, and T. Shibuya: Zool. Sci. 9 (1992) 515. https://doi.org/10.34425/zs000983

34 J. Y. Chew and D. Kurabayashi: J. Bionic Eng. 11 (2014) 268. https://doi.org/10.1016/s1672-6529(14)60036-6

35 S. Shigaki, T. Sakurai, N. Ando, D. Kurabayashi, and R. Kanzaki: IEEE Rob. Autom. Lett. 3 (2018) 76. https:// doi.org/10.1109/1ra.2017.2730361

36 J. Horibe, N. Ando, and R. Kanzaki: Biomimetic and Biohybrid Systems, U. Martinez-Hernandez, V. Vouloutsi, A. Mura, M. Mangan, M. Asada, Y. Prescott and P. Verschure, Eds. (Springer, Cham, 2019) p. 183. https://doi.org/10.1007/978-3-030-24741-6_16

37 N. Ando, S. Emoto, and R. Kanzaki: J. Visualized Exp. 118 (2016) e54802. https://doi.org/10.3791/54802

38 J. Murlis and C. D. Jones: Physiol. Entomol. 6 (1981) 71. https://doi.org/10.1111/j.1365-3032.1981.tb00262.x

39 F. Pedregosa, G. Varoquaux, A. Gramfort, V. Michel, B. Thirion, O. Grisel, M. Blondel, P. Prettenhofer, R. Weiss, V. Dubourg, J. Vanderplas, A. Passos, D. Cournapeau, M. Brucher, M. Perrot, E. Duchesnay, and G. Louppe: J. Mach. Learn. Res. 12 (2012).

40 P. Pansopha, N. Ando, and R. Kanzaki: J. Exp. Biol. 217 (2014) 1811. https://doi.org/10.1242/jeb.090266

41 A. T. Hayes, A. Martinoli, and R. Goodman: IEEE Sens. J. 2 (2002) 260. https://doi.org/10.1109/ JSEN.2002.800682

42 R. A. Russell: Proc. Australian Conf. Robotics and Automation (ACRA, 2003) 1-6.

43 N. Ando and R. Kanzaki: J. Exp. Biol. 218 (2015) 3845. https://doi.org/10.1242/jeb.124834

44 S. Shigaki, M. R. Fikri, and D. Kurabayashi: Sensors 18 (2018) 3720. https://doi.org/10.3390/s18113720

45 Y. Kusuda, Z. Yang, T. Soeda, F. Sassa, and K. Hayashi: Proc. 2019 IEEE Sensors (IEEE, 2019) 1-4. https://doi. org/10.1109/SENSORS43011.2019.8956599

46 N. Misawa, S. Fujii, K. Kamiya, T. Osaki, T. Takaku, Y. Takahashi, and S. Takeuchi: ACS Sens. 4 (2019) 711. https://doi.org/10.1021/acssensors.8b01615

47 P. J. Rodacy, S. Bender, J. Bromenshenk, C. Henderson, and G. Bender: Proc. SPIE Int. Soc. Opt. Eng. (SPIE, 2002) 4742. https://doi.org/10.1117/12.479119

48 N. Ando, S. Emoto, and R. Kanzaki: Bioinspir. Biomim. 8 (2013) 016008. https://doi.org/10.1088/17483182/8/1/016008

49 K. E. Kaissling: Orientation and Communication in Arthropods (Birkhäuser, Basel, 1997) pp. 343-374. https:// doi.org/10.1007/978-3-0348-8878-3 12

50 F. van Breugel and M. H. Dickinson: Curr. Biol. 24 (2014) 274. https://doi.org/10.1016/j.cub.2013.12.023

51 D. Saha, D. Mehta, E. Altan, R. Chandak, M. Traner, R. Lo, P. Gupta, S. Singamaneni, S. Chakrabartty, and B. Raman: Biosens. Bioelectron.: X 6 (2020) 100050. https://doi.org/10.1016/j.biosx.2020.100050

52 T. Sakurai, H. Mitsuno, S. S. Haupt, K. Uchino, F. Yokohari, T. Nishioka, I. Kobayashi, H. Sezutsu, T. Tamura, and R. Kanzaki: PLOS Genet. 7 (2011) e1002115. https://doi.org/10.1371/journal.pgen.1002115 